PUBLICATIONS DE L'INSTITUT MATHÉMATIQUE

Nouvelle série, tome 76(90) (2004), 25-30

\title{
STAR COMPLEMENTS AND MAXIMAL EXCEPTIONAL GRAPHS
}

\section{P. Rowlinson}

Communicated by Slobodan Simić

\begin{abstract}
If $G$ is a maximal exceptional graph then either (a) $G$ is the cone over a graph switching-equivalent to the line graph $L\left(K_{8}\right)$ or (b) $G$ has $K_{8}$ as a star complement for the eigenvalue -2 (or both). In case (b) it is shown how $G$ can be constructed from $K_{8}$ using intersecting families of 3-sets.
\end{abstract}

\section{Introduction}

A finite graph is said to be exceptional if it is connected, has least eigenvalue $\lambda_{\min } \geqslant-2$ and is not a generalized line graph. It was proved by Cameron, Goethals, Seidel and Shult [2] in 1976 that an exceptional graph is representable in the root system $E_{8}$ and hence has at most 36 vertices, each of degree at most 28 (see also [1, Chapter 3] or [3, Chapter 3]). The problem of describing the exceptional graphs remained open until recently, when the maximal exceptional graphs were found using a computer implementation of the star complement technique described in Section 2 [5]. (An exceptional graph is said to be maximal if it is not a proper induced subgraph of another exceptional graph.)

Each exceptional graph is an induced subgraph of (at least) one maximal exceptional graph, and the purpose of this note is to show how all the maximal exceptional graphs can be constructed from $K_{8}$.

The cone over a graph $G$ is the graph $K_{1} \nabla G$ obtained from $G$ by adding a vertex adjacent to all vertices of $G$. It is convenient to partition the maximal exceptional graphs into three types: (a) cones of order 29, (b) graphs of order greater than 29 with a vertex of degree 28, (c) graphs with maximal degree less than 28. There are 430 maximal exceptional graphs of type (a), 37 of type (b) and 6 of type (c). It has already been noted in [5] that those of type (a) are cones over graphs switching-equivalent to the line graph $L\left(K_{8}\right)$, and so we concentrate our attention

2000 Mathematics Subject Classification: Primary 05C50.

Key words and phrases: exceptional graph, eigenvalue, star complement. 
ROWLINSON

on the graphs of types (b) and (c). A construction for the graphs of type (b), independent of star complements, is described in $[\mathbf{5 , 6}]$. A computer-free construction of the maximal exceptional graphs of type (c) is given in [9], along with explicit representations in $E_{8}$ of the six graphs concerned.

\section{Star complements}

Let $G$ be a finite graph with $\mu$ as an eigenvalue of multiplicity $k$. A star set for $\mu$ in $G$ is a set $X$ of $k$ vertices such that $\mu$ is not an eigenvalue of $G-X$, the subgraph induced by the complement $\bar{X}$ of $X$. The subgraph $G-X$ is called a star complement for $\mu$ in $G$. Star sets and star complements exist for any eigenvalue of any graph: proofs of this and the related results which follow can be found in [7, Chapter 7]. If $\mu \neq-1$ or 0 then the $H$-neighbourhoods of vertices in $X$ are non-empty and distinct; in particular, there are only finitely many graphs with a prescribed star complement for $\mu$. The fundamental result is the following, known as the Reconstruction Theorem and its converse.

Theorem 2.1. Let $X$ be a star set for $\mu$ in the graph $G$. If $G-\bar{X}$ and $G-X$ have adjacency matrices $A_{X}$ and $C$ respectively then $G$ has an adjacency matrix of the form $\left(\begin{array}{cc}A_{X} & B^{T} \\ B & C\end{array}\right)$, where

$$
\mu I-A_{X}=B^{T}(\mu I-C)^{-1} B .
$$

Conversely, if (1) holds and $A_{X}$ has size $k \times k$, then the null space of $\mu I-A$ consists of the vectors $\left(\begin{array}{c}\mathbf{x} \\ (\mu I-C)^{-1} B \mathbf{x}\end{array}\right)$, where $\mathbf{x} \in \mathbb{R}^{k}$.

If we denote the columns of $B$ by $\mathbf{b}_{u}(u \in X)$ and equate matrix entries in (1) then we obtain the following.

COROLlary 1. With the notation above, $X$ is a star set for $\mu$ if and only if:

$$
\mathbf{b}_{u}^{T}(\mu I-C)^{-1} \mathbf{b}_{u}=\mu, \quad \text { for all } u \in X
$$

and

$$
\mathbf{b}_{u}^{T}(\mu I-C)^{-1} \mathbf{b}_{v} \in\{-1,0\}, \quad \text { for all distinct } u, v \in X .
$$

\section{Exceptional graphs}

It is shown in [8] that a graph with $\lambda_{\min }=-2$ is exceptional if and only if it has an exceptional star complement for -2 . By the Interlacing Theorem for eigenvalues [12, Theorem 34.2.2], such a star complement has least eigenvalue greater than -2 . Now the connected graphs with $\lambda_{\min }>-2$ are given by the following theorem of Doob and Cvetković $[\mathbf{1 0}]$. The notation $L\left(G ; a_{1}, \ldots, a_{n}\right)$ for a generalized line graph is explained in [3, Chapter 3$]$. 
THEOREM 3.1. If $H$ is a connected graph with least eigenvalue greater than -2 , then one of the following holds:

(i) $H=L(T ; 1,0, \ldots, 0)$ where $T$ is a tree;

(ii) $H=L(K)$ where $K$ is a tree or a unicyclic graph with an odd cycle;

(iii) $H$ is one of 20 graphs on 6 vertices representable in $E_{6}$;

(iv) $H$ is one of 110 graphs on 7 vertices representable in $E_{7}$ (but not $E_{6}$ );

(v) $H$ is one of 443 graphs on 8 vertices representable in $E_{8}$ (but not $E_{7}$ ).

This result was obtained independently of root systems, and since there are only finitely many graphs with a prescribed star complement for -2 , we have here an alternative proof that there are only finitely many exceptional graphs. There are 573 exceptional graphs with least eigenvalue greater than -2 ; moreover, if $G$ is a maximal exceptional graph, then a star set for -2 is maximal and, as noted in [5], the corresponding star complement is one of the 443 exceptional graphs from Theorem 3.1(v). Accordingly, the maximal exceptional graphs were determined by taking each of these 443 graphs in turn as a star complement for -2 , and finding the maximal sets of vectors $\left\{\mathbf{b}_{u}: u \in X\right\}$ that satisfy equations (2) and (3) in Corollary 2.2 (cf. [11, Algorithm 2.4]). Details appear in $[\mathbf{6}]$; the results were announced in [5], where the star complements are labelled E001 to E443 and the maximal exceptional graphs are labelled M001 to M473. None of the graphs E001, ..., E443 is a star complement common to all of M001, ..., M473, although nine are common to all but one of the maximal exceptional graphs, namely $K_{1} \nabla L\left(K_{8}\right)$. For one of these nine star complements (E440), a computer-free proof of this fact is outlined in [4].

We observe that for an exceptional star complement common to the graphs of type (b) and (c), we may take the graph $H_{8}$ obtained from $K_{7}$ by adding a vertex of degree 5. This is the 'simplest' form for a candidate in that it has a divisor of order 3 (equivalently an equitable partition into three parts). Examples of maximal exceptional graphs constructed from $H_{8}$ as a star complement for -2 are given in [5].

To see that $H_{8}$ is a star complement in each graph of type (c) it suffices to inspect the representations of such graphs in $E_{8}$ given in [9]. For graphs of type (b), the observation follows from [5, Theorem 3.7]: a graph of type (b) has an induced subgraph isomorphic to $\overline{S\left(K_{1,7}\right)}$, where $S\left(K_{1,7}\right)$ denotes the graph obtained from $K_{1,7}$ by subdividing each edge.

Now we turn to non-exceptional star complements. Again, no non-exceptional graph occurs as a star complement for -2 in every maximal exceptional graph. To see this without recourse to a computer note first that the components of a nonexceptional star complement are generalized line graphs of the types described in Theorem 3.1(i)(ii). We can readily identify such induced subgraphs of $K_{1} \nabla L\left(K_{8}\right)$, and show that at least one does not occur in the smallest maximal exceptional graph (M001) or $K_{1} \nabla C$ where $C$ is a Chang graph (graphs M004, M005, M006). However $K_{8}$ is a subgraph of $\overline{S\left(K_{1,7}\right)}$ and hence of each graph of type (b); and it is also a subgraph of each graph of type (c), as we can check directly from [9]. Accordingly we have a computer-free proof of the following result, which shows 
that every maximal exceptional graph can be constructed from $K_{8}$ in (at least) one of two ways.

THEOREM 3.2. If $G$ is a maximal exceptional graph then either (1) $G$ is the cone over a graph switching-equivalent to $L\left(K_{8}\right)$, or $(2) G$ has $K_{8}$ as a star complement for -2 , or both.

It remains to investigate the construction of the maximal exceptional graphs which arise in case (2) of Theorem 3.2. We let $H=G-X \cong K_{8}$ and apply Corollary 2.2 with $\mu=-2$ and $C=J-I$ (where $J$ is the all- 1 matrix of size $8 \times 8$ ). From equation (2), or by direct calculation, we find that the $H$-neighbourhoods $\Delta_{H}(u)(u \in X)$ have size 3 or 6 . Now equation (3) yields the following:

- if $\left|\Delta_{H}(u)\right|=\left|\Delta_{H}(v)\right|=3$, then either $\left|\Delta_{H}(u) \cap \Delta_{H}(v)\right|=2$ and $u \sim v$ (' $u$ is adjacent to $\left.v^{\prime}\right)$ or $\left|\Delta_{H}(u) \cap \Delta_{H}(v)\right|=1$ and $u \nsim v$;

- if $\left|\Delta_{H}(u)\right|=3$ and $\left|\Delta_{H}(v)\right|=6$ then either $\left|\Delta_{H}(u) \cap \Delta_{H}(v)\right|=3$ and $u \sim v$ or $\left|\Delta_{H}(u) \cap \Delta_{H}(v)\right|=2$ and $u \nsim v$;

- if $\left|\Delta_{H}(u)\right|=\left|\Delta_{H}(v)\right|=6$ then either $\left|\Delta_{H}(u) \cap \Delta_{H}(v)\right|=5$ and $u \sim v$ or $\left|\Delta_{H}(u) \cap \Delta_{H}(v)\right|=4$ and $u \nsim v$.

Since any two 6-subsets of an 8-set intersect in at least 4 elements, the necessary and sufficient conditions on the family of $H$-neighbourhoods (of size 3 or 6 ) are (i) any two sets of size 3 intersect, (ii) a set of size 3 intersects a set of size 6 in at least 2 elements.

Now let $\mathcal{F}$ be a family of 3 -subsets of $\{1,2, \ldots, 8\}$, and let $\mathcal{F}^{(2)}$ be the family of 2 -sets which are contained in some 3 -set of $\mathcal{F}$. We say that $\mathcal{F}$ is an intersecting family if $U \cap V \neq \emptyset$ for all $U, V \in \mathcal{F}$; and such a family $\mathcal{F}$ is complete if there does not exist an intersecting family of 3-sets $\mathcal{F}_{0}$ such that $\mathcal{F} \subset \mathcal{F}_{0}$ and $\mathcal{F}^{(2)}=\mathcal{F}_{0}^{(2)}$. (For example, if $\mathcal{F}=\{138,157,568\}$ then $\mathcal{F}$ is not complete because we can take $\mathcal{F}_{0}=\mathcal{F} \cup\{158\}$.) The next result shows that a maximal exceptional graph with $K_{8}$ as a star complement for -2 is determined by a complete intersecting family of 3 -subsets of $\{1,2, \ldots, 8\}$, and vice versa. Here we take $V(H)=\{1,2, \ldots, 8\}$ and write $\overline{i j}$ for the complement of $\{i, j\}$ in $V(H)$.

THEOREM 3.3. Let $G$ be a graph with $K_{8}$ as a star complement for -2 , say $H=G-X \cong K_{8}$. Then $G$ is a maximal exceptional graph if and only if the family of $H$-neighbourhoods $\Delta_{H}(u)(u \in X)$ has the form $\mathcal{F}_{3} \cup \mathcal{F}_{6}$ where $\mathcal{F}_{3}$ is a complete intersecting family of 3-sets and $\mathcal{F}_{6}=\left\{\overline{i j}: i j \notin \mathcal{F}_{3}^{(2)}\right\}$.

Proof. First suppose that $G$ is a maximal exceptional graph, and let $\mathcal{F}_{3}$ be the family of $H$-neighbourhoods of size 3 . From the remarks above we know that $\mathcal{F}_{3}$ is an intersecting family. If $i j \notin \mathcal{F}_{3}^{(2)}$ then the 6 -set $\overline{i j}$ intersects each member of $\mathcal{F}_{3}$ in at least 2 elements. Now the maximality of $X$ ensures first that the $H$-neighbourhoods include every such 6 -set, and secondly that $\mathcal{F}_{3}$ is complete. 
Conversely, if the family of $H$-neighbourhoods has the form given then $X$, and hence $G$, is maximal. Moreover $G$ is exceptional because a graph obtained from $K_{8}$ by adding a vertex of degree 3 or 6 is itself exceptional.

Note that if $\mathcal{F}_{3}=\emptyset$ then $\mathcal{F}_{6}$ consists of all 286 -sets. In this case $G$ is the unique maximal exceptional graph of order 36, denoted by M473 in [5]. It is straightforward to show that if we switch $G$ with respect to $V(H)$ then we obtain $L\left(K_{9}\right)$. The remaining examples given here provide an independent means of constructing the six maximal exceptional graphs of type (c).

\section{Examples}

(i) For M001 (of order 22), we may take $\mathcal{F}_{3}$ to consist of lines in the projective plane $P G(3,2)$ whose points are $1,2, \ldots, 7$; and then $\mathcal{F}_{6}$ consists of all 6 -subsets of $\{1,2, \ldots, 7\}$.

(ii) For M002 (of order 28), we may take $\mathcal{F}_{3}=\{567,568,578,678,156,178,257,268,358,367\}$; and then $\mathcal{F}_{6}=\{\overline{12}, \overline{13}, \overline{14}, \overline{23}, \overline{24}, \overline{34}, \overline{45}, \overline{46}, \overline{47}, \overline{48}\}$

(iii) For M417 (of order 29), we may take $\mathcal{F}_{3}=\{128,148,157,158,358,568\}$.

(iv) For M428 (of order 29), we may take $\mathcal{F}_{3}=\{128,138,148,157,158,568\}$.

(v) For M437 (of order 30), we may take $\mathcal{F}_{3}=\{128,138,157,158,568\}$.

(vi) For M462 (of order 31), we may take $\mathcal{F}_{3}=\{138,157,158,568\}$.

M. Lepović (personal communication) has shown by computer that exactly 363 maximal graphs have $K_{8}$ as a star complement for -2 ; in particular, there are exactly 320 maximal exceptional graphs which satisfy both condition (1) and condition (2) in Theorem 3.2.

\section{References}

[1] A.E. Brouwer, A.M. Cohen and A. Neumaier, Distance-Regular Graphs, Springer-Verlag, Berlin, 1989

[2] P. J. Cameron, J. M. Goethals, J. J. Seidel and E. E. Shult, Line graphs, root systems, and elliptic geometry, J. Algebra 43 (1976), 305-327.

[3] P. J. Cameron and J.H. van Lint, Designs, Graphs, Codes and their Links, Cambridge University Press, 1991.

[4] D. Cvetković, Graphs with least eigenvalue -2; a historical survey and recent developments in maximal exceptional graphs, Linear Alg. Appl. 356 (2002), 189-210.

[5] D. Cvetković, M. Lepović, P. Rowlinson and S. K. Simić, The maximal exceptional graphs, J. Combin. Theory Ser. B 86 (2002), 347-363.

[6] D. Cvetković, M. Lepović, P. Rowlinson and S. K. Simić, Computer investigations of the maximal exceptional graphs, Technical Report CSM-160, Department of Computing Science and Mathematics, University of Stirling, 2001.

[7] D. Cvetković, P. Rowlinson and S. K. Simić, Eigenspaces of Graphs, Cambridge University Press, 1997.

[8] D. Cvetković, P. Rowlinson and S.K. Simić, Graphs with least eigenvalue -2: the star complement technique, J. Algebraic Combinatorics 14 (2001), 5-16.

[9] D. Cvetković, P. Rowlinson and S. K. Simić, The maximal exceptional graphs with maximal degree less than 28, Bull. Serb. Acad. Sci. Arts 22:26 (2001), 115-131. 
[10] M. Doob and D. Cvetković, On spectral characterizations and embedding of graphs, Linear Alg. Appl. 27 (1979), 17-26.

[11] M. N. Ellingham, Basic subgraphs and graph spectra, Australasian J. Combinatorics 8 (1993), 247-265.

[12] V. V. Prasolov, Problems and Theorems in Linear Algebra, American Math. Soc., Providence, RI, 1994.

Mathematics and Statistics Group

(Received 1811 2003)

Department of Computing Science and Mathematics University of Stirling

Scotland, FK9 4LA

p.rowlinson@stirling.ac.uk 